
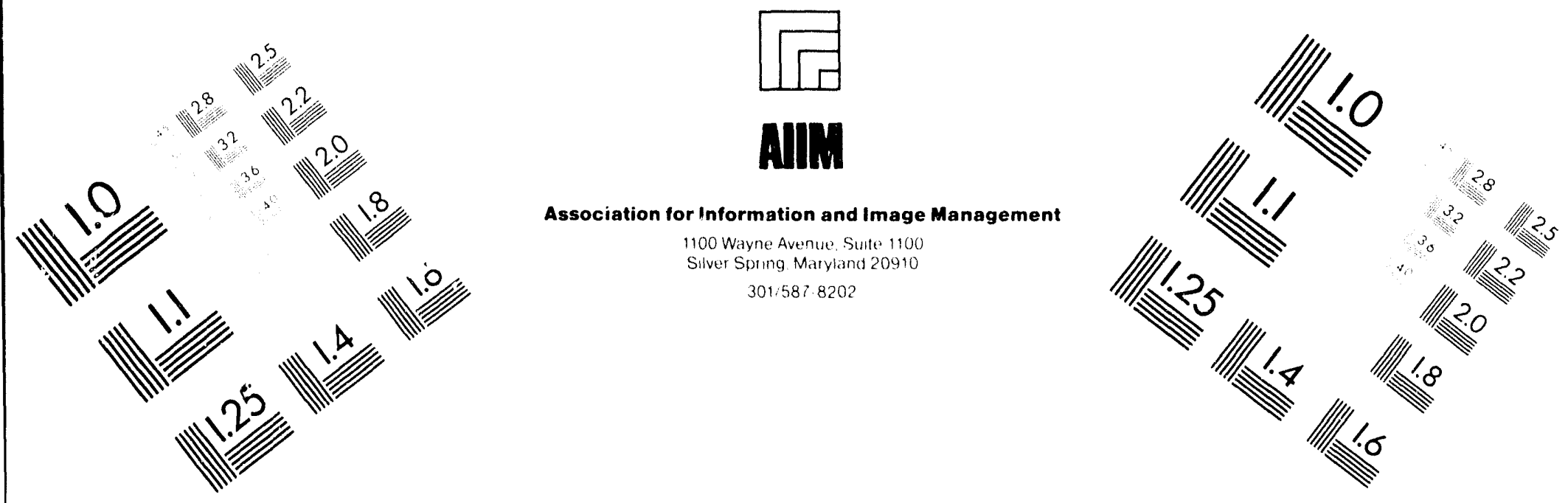

\title{
Centimeter
}

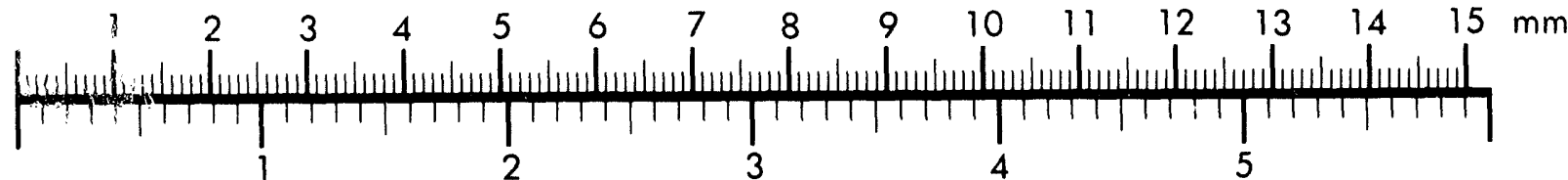

Inches
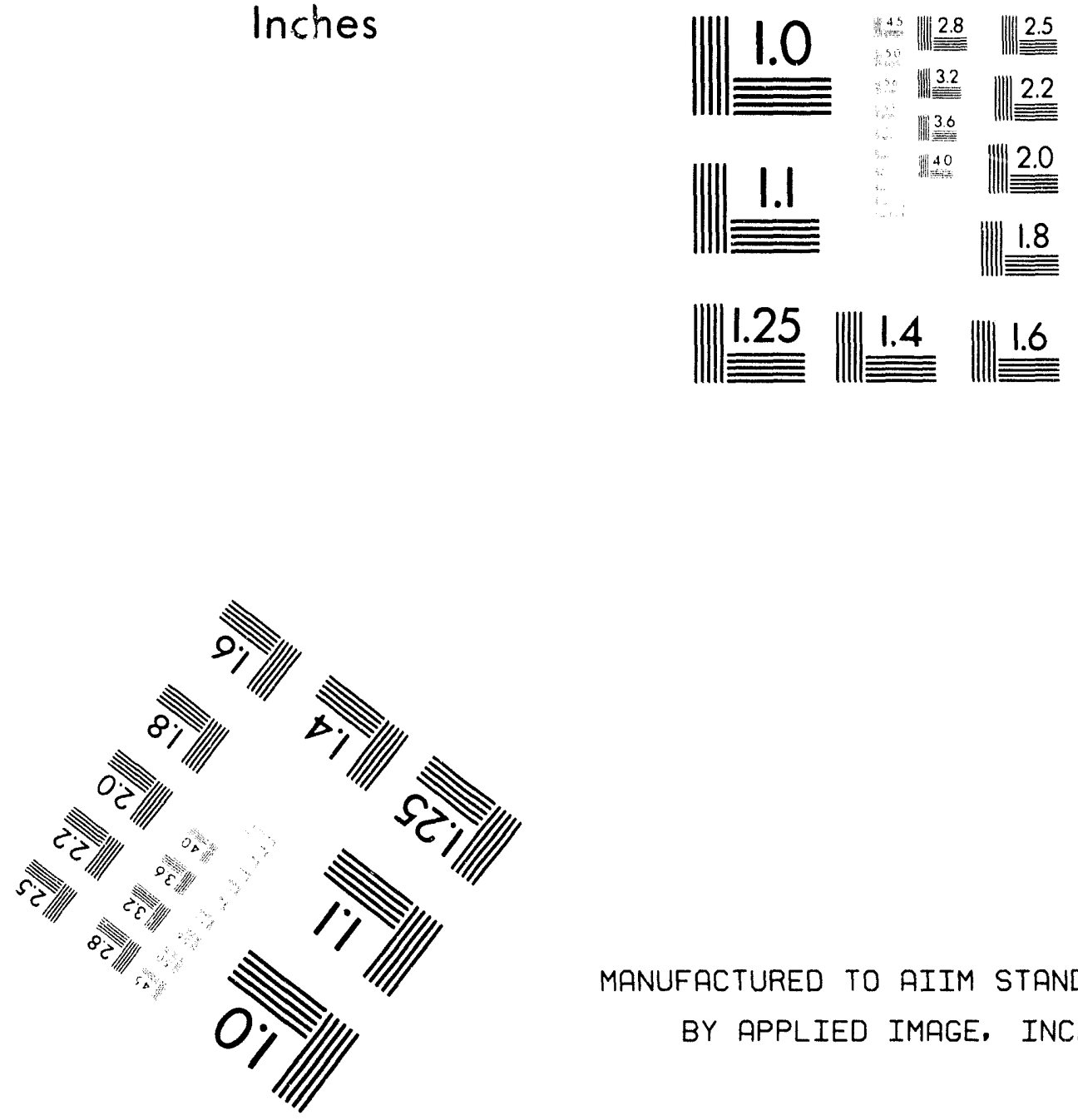

MANUFACTURED TO AIIM STANDARDS

BY APPLIED IMAGE, INC.

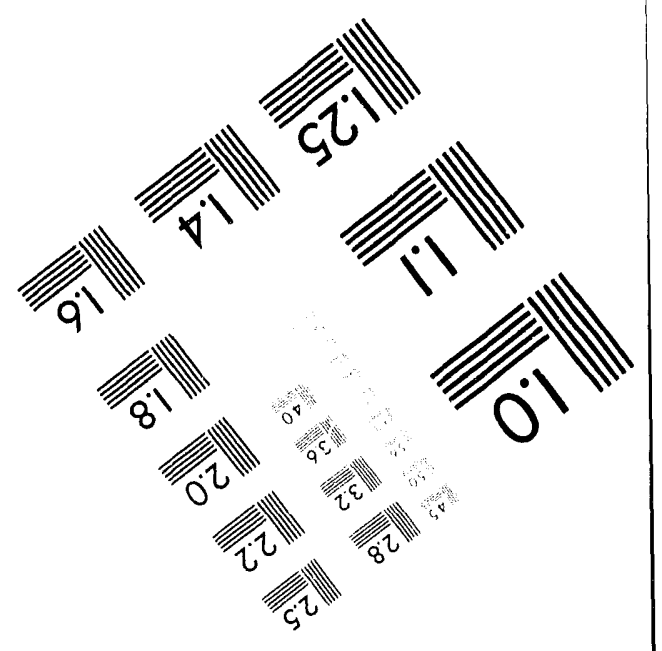



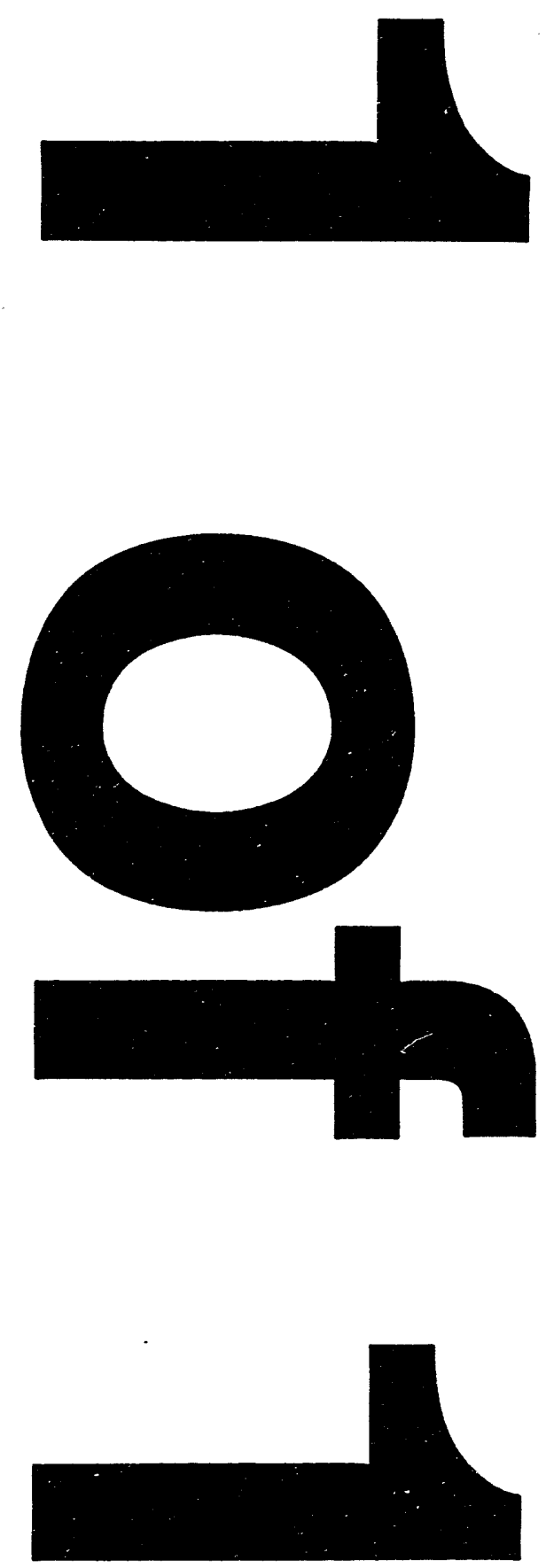
This report was prepared as an account of work sponsored by an agency of the United States Government. Neither the United States Government nor any agency thereof, nor any of their employees, makes any warranty, express or implied, or assumes any legal liability or responsibility for the accuracy, completeness, or usefulness of any information, apparatus, product, or process disclosed, or represents that its use would not infringe privately owned rights. Reference herein to any specific commercial product, process, or seivice by trade name, trademark, manufacturer, or otherwise, does not necessarily constitute or imply its endorsement, recommendation, or favoring by the United States Government or any agency thereof. The views and opinions of authors expressed herein do not necessarily state or reflect those of the United Stales Government or any agency thereof. 


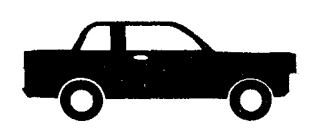

INTRODUCTION

The following is a summary side-by-side comparison of the fleet provisions and incentives under the Clean Air Act Amendments of 1990 (Public Law 101-549) and the Energy Policy Act of 1992 (Public Law 102-486). For more information on how to comply, contact your regional Department of Energy and Environmental Protection Agency support offices in addition to your state energy office.

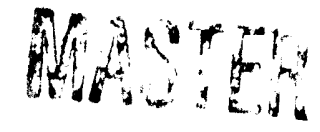




\section{Comparative Alternative/Clean Fuels Provisions of \\ the Clean Aip Act Amendments of 1990 and \\ the Energy Policy Act of 1992}

\begin{tabular}{|c|c|c|}
\hline Provision: & Clean Air Act Amendinents of 1900 & , Energy Policy Act of 1992 \\
\hline $\begin{array}{l}\text { Persons } \\
\text { Affected }\end{array}$ & 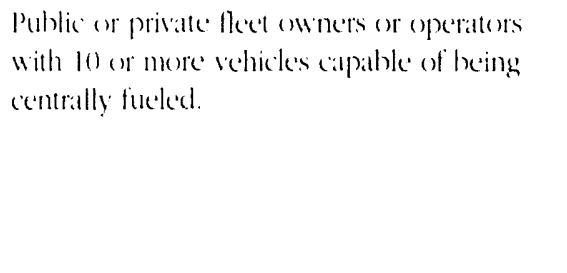 & 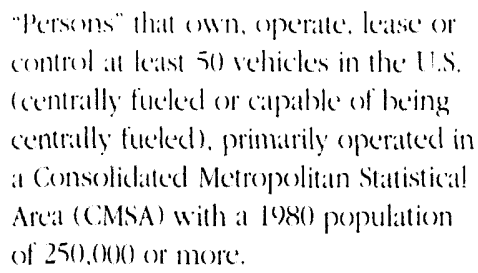 \\
\hline Areas Affected & $\begin{array}{l}22 \text { metropolitan areas with a } 1980 \text { population } \\
\text { of } 250,000 \text { or more and designated by file as } \\
\text { in severe or extreme nonattainment of either } \\
\text { ambient ozone or carbon monoxide (CO) } \\
\text { stanclateds (see attachment A). } \\
\text { Other areas may "opt-in" to the program per } \\
\text { state air guality attainment strategy (no other } \\
\text { areas have done so } 6 \text { date). }\end{array}$ & $\begin{array}{l}\text { Nore than } 120 \text { cities with a } 1980 \\
\text { population of } 250.000 \text { or more } \\
\text { (see attachment } 3 \text { ). }\end{array}$ \\
\hline Fleet Definition & $\begin{array}{l}10 \text { or more heary-duty or light-cluty wehicles } \\
\text { located in the affected area. }\end{array}$ & 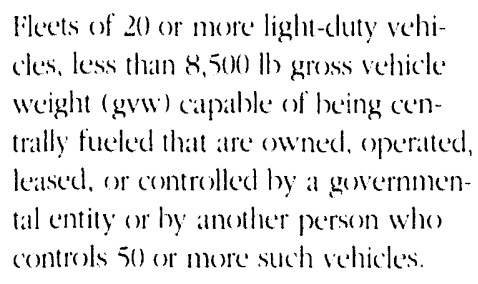 \\
\hline Effective Dates & 1008 (see altachment C.). & $\begin{array}{l}1993 \text { (fecteral flects) } \\
1996 \text { (state flects) } \\
1996 \text { ("fuel provider") } \\
1999 \text { (municipal/private) } \\
2002 \text { (municipal/private by } 1 \text { )( )E rule) } \\
\text { (see attachment c). }\end{array}$ \\
\hline $\begin{array}{c}\text { Purchase } \\
\text { Requirements }\end{array}$ & $\begin{array}{l}\text { Clean Fuel Vehicke (CFV) Fleed Program } \\
\text { (vehicles under } 26,600 \text { Ih) } \\
\text { (sece attachment C). }\end{array}$ & $\begin{array}{l}\text { There are requirements for federal, } \\
\text { state, fuel provider, municipal and } \\
\text { private flects (see attachment (c). }\end{array}$ \\
\hline Fuel Definition & 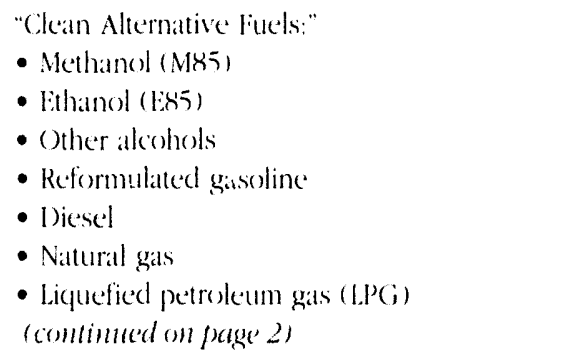 & 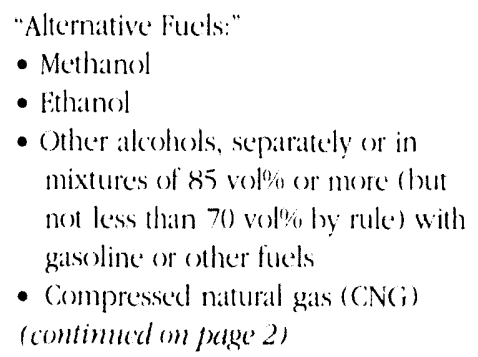 \\
\hline
\end{tabular}




\begin{tabular}{|c|c|c|}
\hline Provision & Clean Air Act Amendinents of 1990 & Energy Policy Act of 1992 \\
\hline 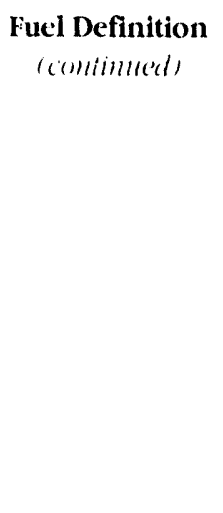 & 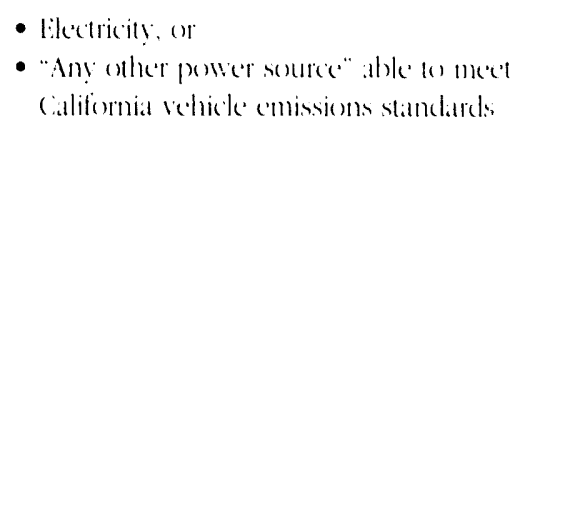 & 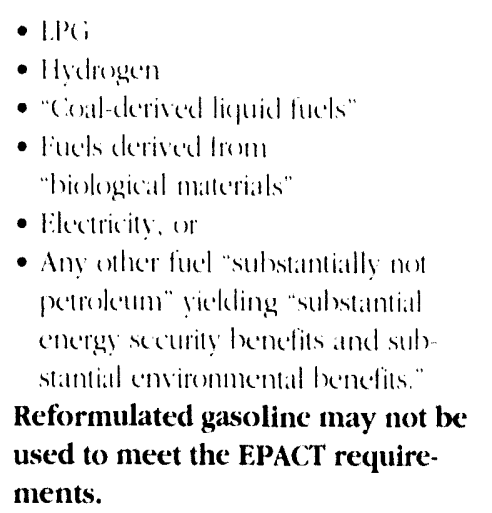 \\
\hline Credits & 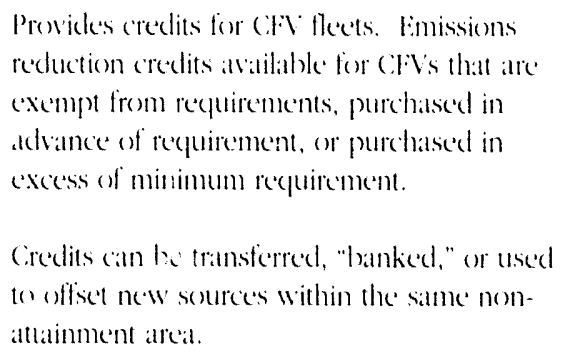 & 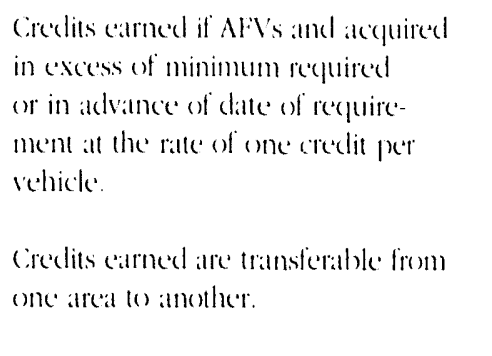 \\
\hline Buses & 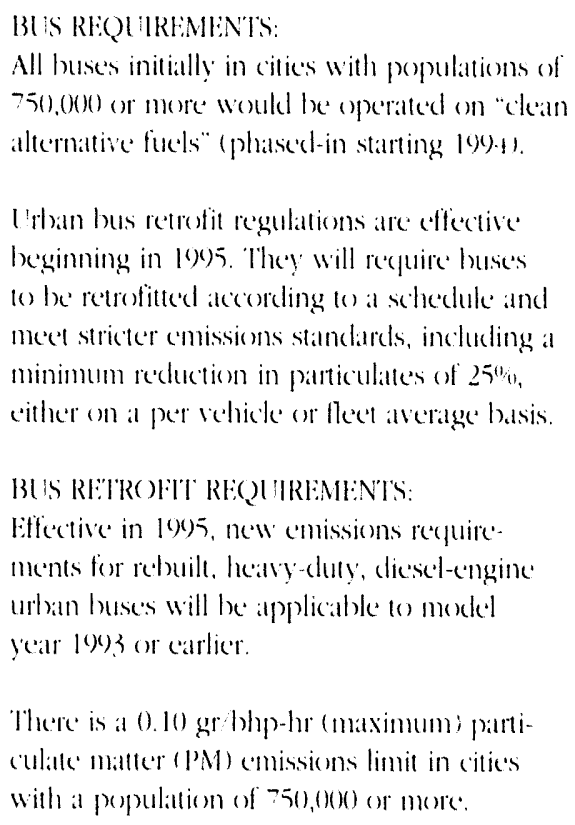 & 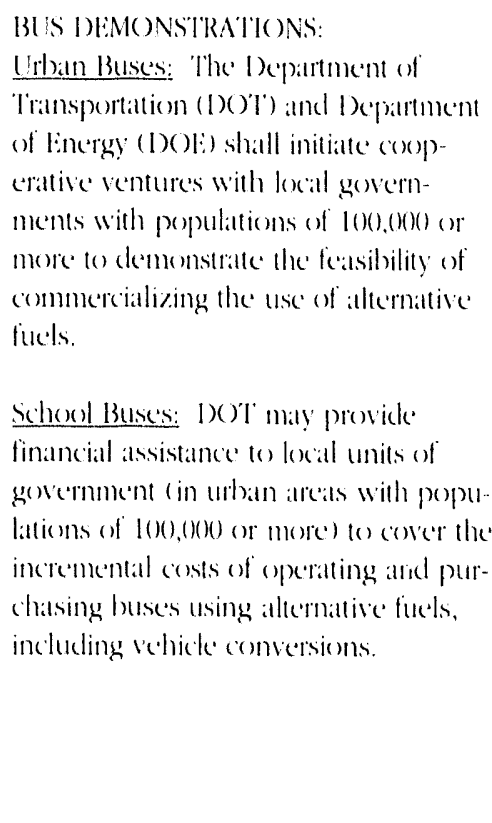 \\
\hline $\begin{array}{l}\text { Non-Road } \\
\text { Engines }\end{array}$ & 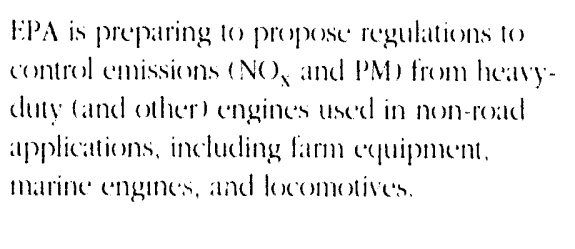 & 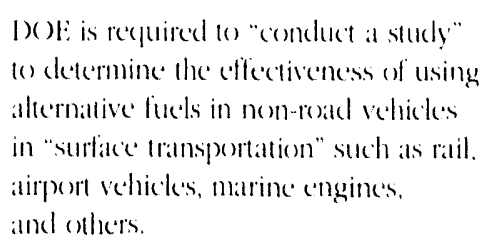 \\
\hline
\end{tabular}




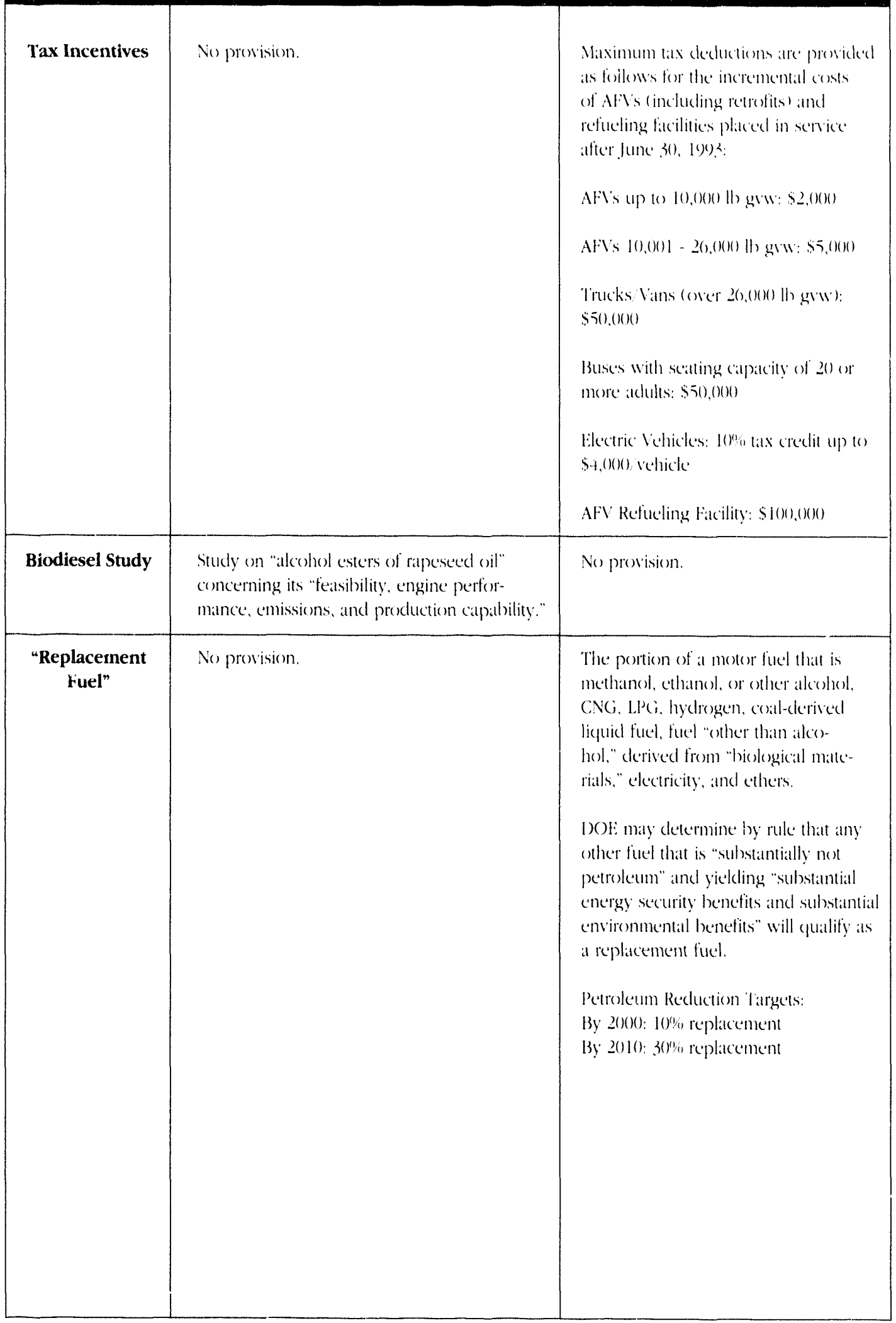




\title{
Altachment A \\ Areas Covered in Clean Air Act Amendments of 1990 \\ (Clean-Fuel Vehicle Fleet Program)
}

\author{
Allanta, (i)
}

Baltimore, MII)

Bitton Rouge, I.A

Beatumont-Pont Arthur, IX'

Beston-Latwrence-Worcester, MA-NH

Chicag(e-Cally-lake county, II-IN-WI

Denver-Boulder, (c)

lil Pats(), IX

Circiter connecticut, cll

Houston-Galeseston-Brazoria, IX

Los Angeles-Anaherim-Riverside, (A

Milwatukere-Racine, Wl

New York-Long lsland-Northern Jersey, NY-N!

Philadelphia-Wilmington-Trenton, PA-I)E-MI)-N!

Providence-Pawtucket-Fall River, RI-MA

Satramento, (A)

San Diego, (A

San foacuin Valley. CA

Southeast Desert, CA

Springficeld, MA

Ventura County, CA

Washingt(m, D).(., MI)-VA-1)(: 


\section{Altachment B \\ Metropolitan Areas with a 1980 Population of 250,000 or More}

- Allany-Schenectidy-Troy, NY

- Alluquerque, NM

- Allentown-Bethlehem-Kaston, PA-N]

- Appleton-Oshkosh-Veenah, WI

- Atlantí cia

- Allantic City, NJ

- Augustia (ia

- Austin, TX

- Baticerslield, ca

- Battimore, MD

- Baton Rouge, La

- Besilumont-Port Arthur, TX

- Binghamton, NY

- Birmingham, AL

- Bosion-latwrence-Salem, MA-NH

- Buffato-Niagra Falls, NY

- Canton, OH

- Charleston, SC

- Charleston. WV

- Charlotte-Castonia-Rock Hill. $\mathrm{NC}-\mathrm{SC}$

- Chatianooga, IN-CiA

- Chicago-Gary-lake County, IL-IN-W]

- Cincinnati-Hamilton, OH-KY-IN

- Cleveland-Akron-Lorain, (OH

- Colorado Springs, CO

- Columbia, SC

- Corpus Christi, TX

- Dallas-Fort Wurth, TX

- Davenport-Rock Island-Moline. IA-IL

- Dayton-Springfield, OH

- Daytona Beach, Fl.

- Denver-Boulder, CO

- Des Moines, la

- Detroit-Ann Arbor, MI

- Duluth, MN-WI

- El Paso, TX

- Erie, PA

- Eugene-Springfiedd. OR

- Evansville, IN-KY

- Flint, MI

- Fort Wayne, IN

- Fresno, CA

- Grand Rapids, MI
- Greenshoro-Winston Sialem-fligh Point, NC:

- Circenville-spartanhurg, SC:

- Harrisburg-lebanon-Carlisle, PA

- Hartford-New BritainMiddletown. C.'P

- Honolula, HI

- Houston-Cialseston-Brazoria, TX

- Huntinglon-Ashland. WV-K'-OH

- Indiamapolis, is

- Jackion, MS

- facksomville, fil.

- Johnson City-Kingsport-Bristol, TN-VA

- Johnstown, PA

- Kansas (ity. MO-KS

- Knoxville, TN

- Lakeland-Winter Haven, FL.

- Lancilster. PA

- Lansing-East Lansing. MI

- Las Vegals, NV

- Lexington-Fayette, KY'

- Little Rock-North Little Rock, AR

- Los Angeles-AnaheimRiverside. C.A

- Louisville, KY-IN

- Macon-Warner Rohins, (iA

- Madison, WI

- McAllen-Edinburg-Mission. IX

- Melbourne-Titusville-Palm Bay, Fl

- Memphis, TN-AR-MS

- Miami-iont Lauderdale, Fl.

- Milwalukee-Racine, WI

- Minneapolis-St, Paul, MN-WI

- Mobile, AL

- Modesto, CA

- Montgomery, AL

- Nashville, TN

- New Haven-Muridien, CI

- New London-Norwich, CT-RI

- New Orleans, la

- New York-N. New Jersey-long Island, NY-NJ-C.T

- Norfolk-Virginia Beach-Newport News, va

- Oklahoma Cily, OK
- ()millia, NI:-IA

- orlandor. ri.

- Pensiacolia fil.

- Peoriar Il.

- Philadephlia-Wilmingen-Trenton, Pa-N-(1)(:-M!)

- Pherenix, $\mathrm{d} \%$

- Piltshurgh-Bealrer Vialley, Pa

- Portland-Vancourcer, oR-WA

- Providence-Pantucket-rall River. RI-MA

- Ralcigh-1)urliam. NC

- Reacling. PA

- Richmond-pelershurg. ia

- Rochesiter, Ni

- Rocktord, Il.

- Sitcramente, CA

- Saginaw-Bady City-Midland, MI

- Saint Louis, Mo-ll.

- Salinats-Seaside-Nonterey, CA

- Silt Lake City-()gdkn. IT'

- Sian Antonio, TX

- San Diego, (iA

- San Francisco-()akiland-Sin Jose, CA

- Sitnta Barbara-Sianta Marialompor, CA

- Scranton-Wilkes-Barre, PA

- Séattle-Tacoma, WA

- Shreveport, LA

- Spokanc, WA

- springfiede, MA

- Strocktom, CA

- Syracusc, NY

- Tampa-St. PetersburgClearwater. FL

- Toledo, OH

- Tucson, Az

- Tulsal, OK

- Ulica-Rome, NY

- Washington, I)C-MD)-VA

- West Palm Beach-Buca RatonDelray Beach, Fl.

- Wichita, KS

- Worcester, MA

- York, PA

- Youngstown-IVarren, ()H 


\section{Altactment C \\ A Companiscon of New AFV Purchase Requinements \\ for Affected Vehicles Fleets}

\begin{tabular}{|c|c|c|c|c|c|c|}
\hline \multirow[b]{2}{*}{ léalr } & \multirow{2}{*}{ Clem Ap Act } & \multicolumn{5}{|c|}{ Eneryy Poliny Act } \\
\hline & & $\begin{array}{l}\text { Fecderal } \\
\text { ("\% or Number } \\
\text { of AFVs) }\end{array}$ & $\begin{array}{c}\text { Stitte } \\
(0 \% \text { of } A F V s)\end{array}$ & $\begin{array}{l}\text { Fuel Provider } \\
\text { ("6 of AFVs) }\end{array}$ & $\begin{array}{c}\text { Municipal Private } \\
\text { (carly rule })^{*} \\
\text { (1) of AFVs) }\end{array}$ & $\begin{array}{l}\text { Municipal Privalte } \\
\text { (late rulce } \\
\text { (") of AFVs) }\end{array}$ \\
\hline 1993 & & $5,000 \mathrm{AFV}$ & & & & \\
\hline 1994 & & $7,500 \mathrm{AFVs}$ & & & & \\
\hline 1995 & & $10,000 \mathrm{AFVS}$ & & & & \\
\hline 1996 & & $25 \%$ & $10 \%$ & $30 \%$ & & \\
\hline 1997 & & $33 \%$ & $15 \%$ & $50 \%$ & & \\
\hline 1998 & $30 \%$ & $50 \%$ & $25 \%$ & $70 \%$ & & \\
\hline 1999 & $50 \%$ & $75 \%$ & $50 \%$ & $90 \%$ & $20 \%$ & \\
\hline 2000 & $700^{m}$ & $75 \%$ & $75 \%$ & $90 \%$ & $20 \%$ & \\
\hline 2001 & $100 \%$ & $75 \%$ & $75 \%$ & $90 \%$ & $20 \%$ & \\
\hline 2002 & $100 \%$ & $75 \%$ & $75 \%$ & $90 \%$ & $30 \%$ & $20 \%$ \\
\hline 2003 & $100 \%$ & $75 \%$ & $75 \%$ & $90 \%$ & $40 \%$ & $40 \%$ \\
\hline 2004 & $100 \%$ & $75 \%$ & $75 \%$ & $90 \%$ & $50 \%$ & $60 \%$ \\
\hline 20015 & $100 \%$ & $75 \%$ & $75 \%$ & $90 \%$ & $60 \%$ & $70 \%$ \\
\hline 2006 & $100 \%$ & $75 \%$ & $75 \%$ & $90 \%$ & $70 \%$ & $70 \%$ \\
\hline
\end{tabular}

- Under the Enery. Policy Act the U.S. Energl' Secretary bas tuv opportunities to mele on allernative fitel vebicle purchases for private fleets. If a rulemaking is issued by December 15, 1996, then the percentages in the "early rule" column apply. If a milemaking is not issued until later, (Jamuary 1, 2000 deadline), then the percentages in the "late" mle" column apply. 

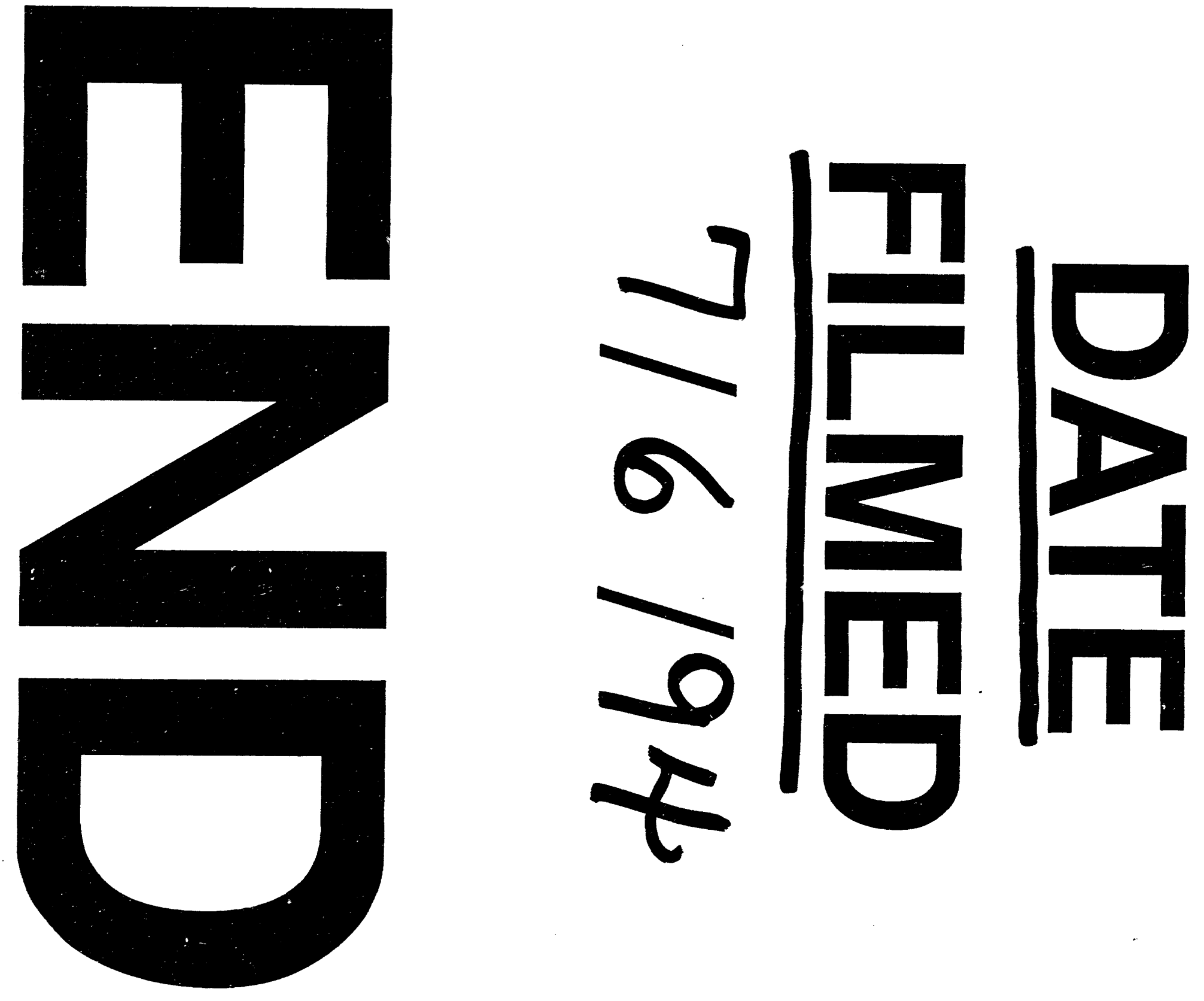
